\title{
Support Region of Semilogarithmic Quantizer for Laplacian Source
}

\author{
Zoran H. Peric ${ }^{1}$, Sinisa B. Suzic ${ }^{2}$, Tijana V. Delic ${ }^{2}$, Nikola Simic ${ }^{1}$ \\ ${ }^{1}$ Department of Telecommunications, Faculty of Electronic Engineering, University of Nis, \\ Aleksandra Medvedeva 14, 18000 Nis, Serbia \\ ${ }^{2}$ Faculty of Technical Sciences, University of Novi Sad, \\ Dositej Obradovic Square: 6, 21000 Novi Sad, Serbia \\ zoran.peric@elfak.ni.ac.rs
}

\begin{abstract}
The overall distortion of semilogarithmic $A$-law quantizer is influenced by its support region, as well as parameter $A$. Current solutions require numerical determination of optimal support region and larger processing time, i.e. simple closed-form solutions for finding optimal values of these two parameters are not provided. In this paper, iterative and closed-form solutions for determination of the support region of semilogarithmic $A$-law quantizer for Laplacian source, unite variance and a set of bit-rates $6 \mathrm{bits} / \mathrm{sample}-10 \mathrm{bits} / \mathrm{sample}$ are proposed. The performance of proposed solutions is compared to the optimal numerical results.
\end{abstract}

Index Terms-Support region; Quantizer; Semiloga rithmic $A$-law.

\section{INTRODUCTION}

The constant development and growth of communication networks created a need for further research in the area of speech coding, in order to provide faster and better speech signal transmission quality. Also, compression efficiency is very important while considering energy assumption in the modern systems [1]. Although G.711 standard, which defines Pulse-Code Modulation (PCM), was adopted back in 1972 [2], it was actively amended in 2009 [3].

Companding quantizers represent an important digitization tool, since they are able to achieve near-constant quality of reconstructed signal for a wide range of input signal's variances, when quantizing non-stationary signals. The reconstructed signal quality obtained by using such technique is not very sensitive to changes of statistical parameters of the input signal and this is achieved by introducing logarithmic compression function. However, this idealization is very hard for exact implementation. By now, different modifications of logarithmic function, practically applicable, have been developed. Two solutions have been standardized in G.711: semilogarithmic compressor function, widely known as $A$-law (applied in Europe) and quasilogaritmic compressor function, widely known as $\mu$-law (applied in USA and Japan) [4].

Manuscript received 2 November, 2017; accepted 7 May, 2018.

The research was conducted within the project "Development of Dialogue Systems for Serbian and Other South Slavic Languages" (TR32035), financed by Ministry of Education, Science and Technological Development of Republic of Serbia.
The quality of quantization is measured by calculating distortion value or signal-to-quantization-noise ratio (SQNR). These measures are highly dependent on the number of quantization levels and the width of quanitizer's support region [4], [5].

While the number of quantization levels depends directly on the desired bit-rate, determination of optimal support region is much more complex. The significance of support region for Laplacian source and optimal compandor was already discussed in [6], [7]. Furthermore, analysis and determination of support region for quasilogarithmic quantizers and the same source were presented in [8]. It can be noticed that optimal design is usually performed for the unite variance [8], whereas the more complex solutions incorporate adaptation [9] or dual-stage quantization [10]. Moreover, support region represents an important design parameter of vector quantizers, as well as quantizers exploited for other signal sources such as Gaussian, Rayligh and Gamma.

In this paper, we provide an iterative and a closed-form solutions for support region determination of $A$-law semilogarithmic quantizer for Laplacian source and a set of bit-rates 6 bits/sample-10 bits/sample. It was shown that differences between the proposed solutions for finding optimal parameters and numerically obtained optimal solution are almost negligible.

The paper is organized as follows. In the chapter two, $A$ law quantizer is described and its distortion is presented. The third chapter features the optimal numeric values for the support region threshold, as well as the $A$ constant. In the fourth chapter, the proposed iterative and closed-form solutions are presented and compared with numerical results. Lastly, the conclusions and further research aims are stated.

\section{Design of Semilogarithmic $A$-LAW QuANTIZER}

Semilogaritmic compressor function consists of linear and logarithmic part and it is given with the following equation

$$
c_{A}=\left\{\begin{array}{cc}
\frac{A x}{1+\ln A}, & |x| \leq \frac{x_{\max }}{A}, \\
\frac{x_{\max }}{\ln A}\left(1+\ln \frac{A x}{x_{\max }}\right), & \frac{x_{\max }}{A}<|x|<x_{\max },
\end{array}\right.
$$


Parameter $x_{\max }$ represents maximal allowed amplitude of quantizer, i.e. support region, while parameter $A$ impacts the border between linear and logaritmic parts and its value is adopted to be 87.6 in PCM telephone systems in Europe [4].

In the rest of the paper, it will be assumed that amplitudes of the input signal are represented by Laplacian probability density function (PDF), which is a good representation for speech samples [4]

$$
p(x)=\frac{1}{\sqrt{2} \sigma} e^{-\frac{|x| \sqrt{2}}{\sigma}} .
$$

For simplicity, it will also be assumed that the value of parameter $\sigma$, i.e. standard deviation, is 1 .

The overall distortion is given as a sum of granular and overload distortion

$$
D_{t o t}=D_{g}+D_{o v}
$$

Granular distortion can be represented as the sum of $D_{g 1}$ and $D_{g 2}$ which are calculated by using the following equations:

$$
\begin{aligned}
& D_{g 1}=\frac{2 x_{\max }^{2}(1+\ln (A))^{2}}{3 N^{2} A^{2}} \int_{0}^{x_{\max } / A} \frac{1}{\sqrt{2}} e^{-\sqrt{2} x} \mathrm{~d} x, \\
& D_{g 2}=\frac{2(1+\ln (A))^{2}}{3 N^{2}} \int_{x_{\max } / A}^{x_{\max }} x^{2} \frac{1}{\sqrt{2}} e^{-\sqrt{2} x} \mathrm{~d} x .
\end{aligned}
$$

Solving the equations, the final expressions are:

$$
\begin{gathered}
D_{g 1}=\frac{x_{\max }^{2}(1+\ln (A))^{2}}{3 N^{2} A^{2}}\left(1-e^{\frac{-x_{\max } \sqrt{2}}{A}}\right), \\
D_{g 2}=\frac{(1+\ln (A))^{2}}{3 N^{2} \sqrt{2}} \times \\
\times\left(-e^{-x_{\max } \sqrt{2}}\left(\sqrt{2}+x_{\max }\left(2+\sqrt{2} x_{\max }\right)\right)+\right. \\
\left.+e^{\frac{-x_{\max } \sqrt{2}}{A}}\left(\sqrt{2}+\frac{x_{\max }}{A^{2}}\left(2 A+\sqrt{2} x_{\max }\right)\right)\right)
\end{gathered}
$$

Overload distortion is given by the following expressions:

$$
\begin{gathered}
D_{o v}=2 \int_{x_{\max }}^{\infty}\left(x-x_{\max }\right)^{2} p(x) \mathrm{d} x, \\
D_{o v}=e^{-\sqrt{2} x_{\max }} .
\end{gathered}
$$

\section{NUMERICAL RESULTS FOR FINDING OPTIMAL SUPPORT REGION}

Value of the parameter $x_{\max }$ strongly influences the value of total distortion. By increasing it, higher values of granular distortion are obtained, but on the other hand overload distortion decreases. The parameter $A$ has also important impact on the performance of quantizer. Higher values of this parameter ensure better robustness of the quantizer regarding change of variance. However, increase of parameter $A$ leads to decrease of maximum SQNR. Optimal values for these parameters are numerically calculated using MATLAB. It was assumed that parameter $A$ takes integer values between 1 and 100 and parameter $x_{\max }$ takes values from the set $X=\{0.01 * k \mid k=1,2 \ldots 1000\}$. For some fixed number of quantization levels, total distortion is calculated for all combinations of parameters $A$ and $x_{\max }$ using (3), (6), (7) and (9). Values giving smallest distortion are considered as optimal and they are presented in Table I, whereas SQNR is calculated using the following equation

$$
S Q N R=10 \log \frac{\sigma^{2}}{D_{\text {tot }}} .
$$

In Fig. 1, maximal SQNR ratio is shown for a various values of parameter $A$ and the total number of quantization levels $N$, using numerically obtained optimal values of support region and unite variance $\sigma^{2}$.

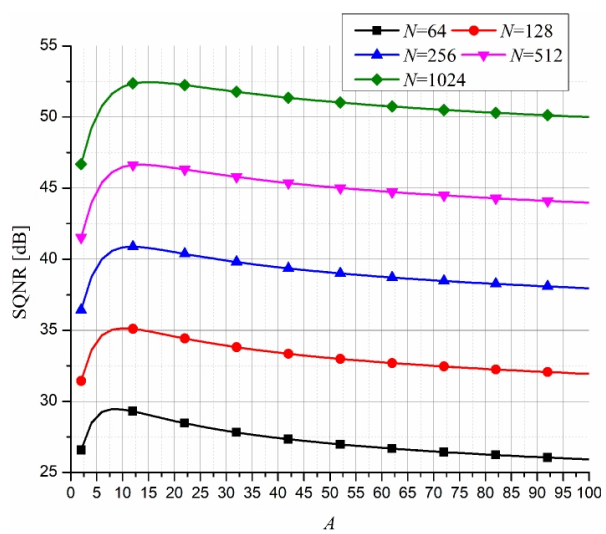

Fig. 1. Maximal SQNR depending on the value of the parameter $A$ for various numbers of quantization levels.

\section{ITERATIVE PROCEDURE FOR FINDING SUPPORT REGION}

The optimal support region value could be determined by finding a point in which partial derivative of the total distortion is equal to zero, i.e. by solving

$$
\frac{d}{d x_{\max }} D_{t o t}=0 .
$$

However, the solution from (11) is not a closed-form. One of the possible general approaches for solving this equation is by finding a suitable iterative function [11]. By solving (11), it can be seen that a few solutions can be formed. However, as the main goal is to provide a simple solution which converges quickly, we propose a solution of a form $x^{(i+1)}=g\left(x^{(i)}\right)$. As closed-form solutions are not provided in the literature, we have decided to analyse determination of optimal support region by applying iterative procedure for the solution obtained from (11)

$$
x_{\max }^{(i+1)}=\frac{1}{\sqrt{2}} \ln \frac{3 N^{2} \sqrt{2}}{(1+\ln A)^{2}\left(\frac{2 x_{\max }^{(i)}}{A^{2}}\left(1-e^{\frac{-\sqrt{2} x_{\max }^{(i)}}{A}}\right)+C_{1}\right)},
$$


where $C_{1}=\sqrt{2}\left(x_{\max }^{(i)}\right)^{2} e^{-\sqrt{2} x_{\max }^{(i)}}$.

Since it could be expected that optimal support value depends on both, the number of quantization levels and the parameter $A$, our goals include determination of optimal initial value for iterative procedure, denoted with $x_{i n}=x_{\max }^{(0)}$, as well as estimation of required number of iterations. For such task, we exploit a stop-criterion defined with

$$
\operatorname{SQNR}\left(x_{\max }^{(i+1)}\right)-\operatorname{SQNR}\left(x_{\max }^{(i)}\right)<5 \times 10^{-3} \mathrm{~dB} .
$$

Fig. 2 shows SQNR for the value of iteratively calculated $x_{\max }$ depending on the number of iterations, for a various values of parameters $N$ and $x_{i n}$. Furthermore, it was chosen the case of $A=20$ considering it as a near-optimal for a discussed set of bit-rates.

By observing Fig. 2, it can be unambiguously seen that for all observed system configurations near-optimal SQNR value is achieved after the first iteration. However, it should be noted that SQNR is strongly dependent on the choice of initial value, $x_{i n}$, of iterative procedure. For values $x_{i n} \geq 8$, near-optimal SQNR is achieved without applying iterative algorithm. This feature is discussed in the rest of the paper in order to provide the closed-form solution defined with following equation

$$
x_{\max }^{\text {proposed }}=\frac{1}{\sqrt{2}} \ln \frac{3 N^{2} A^{2}}{\sqrt{2} x_{i n}(1+\ln A)^{2}\left(1-e^{-\sqrt{2} x_{i n} / A}\right)} .
$$

In the process of proposing the closed-form solution, we have excluded parameter $C_{1}$ from (12), as the results are not highly-dependent.

System performance for initial values of optimal support region, $x_{i n}=8$ and $x_{i n}=10$, is shown in Table I and Table II, respectively.

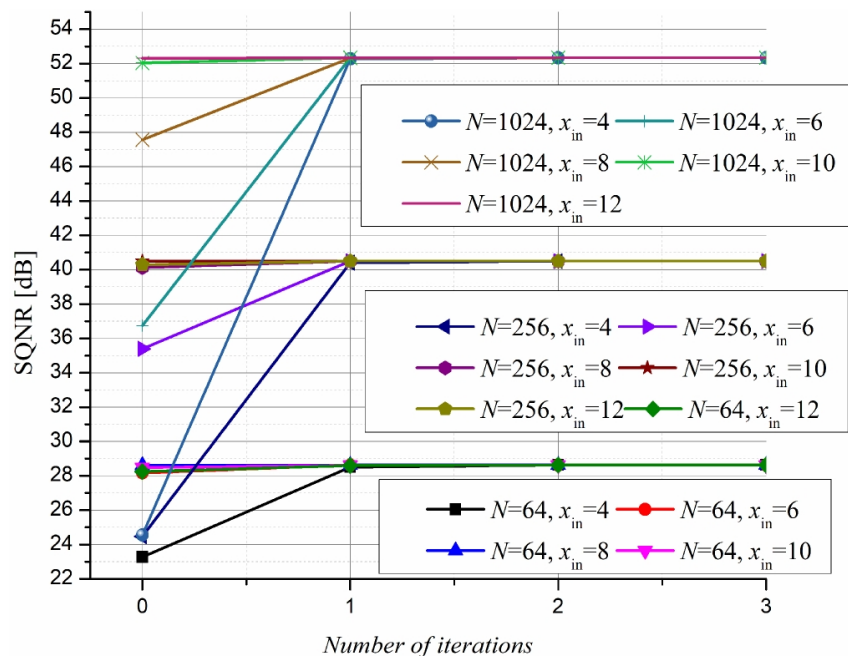

Fig. 2. SQNR for the iteratively calculated support region and various values of initial support range and number of quantization levels.

The system performance is provided for various values of parameter $A$ and number of quantization levels $N$. Besides the performance of the proposed system, we have also shown the results obtained numerically for the optimal support region.

As the second measure of quality, we have observed relative errors between optimal support region and the proposed one, as well as between corresponding values of SQNRs, which are defined as follows:

$$
\begin{gathered}
\delta_{i}^{s p}=\frac{\left|x_{\max i}-x_{\max }\right|}{x_{\max }} \times 100 \%, \\
\delta_{i}^{s q}=\frac{\left|S Q N R_{i}-S Q N R\right|}{S Q N R} \times 100 \%,
\end{gathered}
$$

where $i=1,2$.

In (15) and (16) $i=1$ refers to the iterative procedures whereas $i=2$ refers to the closed-form solutions, while "sp"

\begin{tabular}{|c|c|c|c|c|c|c|c|c|c|c|c|c|}
\hline \multirow[b]{2}{*}{$\mathbf{N}$} & \multirow[b]{2}{*}{$\mathbf{A}$} & \multicolumn{3}{|c|}{ Iterative procedure } & \multicolumn{2}{|c|}{ Closed-form solution } & \multicolumn{2}{|c|}{ Optimal solution } & \multicolumn{2}{|c|}{ Support rel. error } & \multicolumn{2}{|c|}{ SQNR rel. error } \\
\hline & & $x_{\max 1}$ & NoI & $\mathrm{SQNR}_{1}[\mathrm{~dB}]$ & $x_{\max 2}$ & $\mathrm{SQNR}_{2}[\mathrm{~dB}]$ & $x_{\max }$ & SQNR [dB] & $\delta_{1}{ }^{\mathrm{sp}}[\%]$ & $\delta_{2}{ }^{\text {sp }}[\%]$ & $\delta_{1}$ sq $[\%]$ & $\delta_{2}{ }^{\text {sq }}[\%]$ \\
\hline \multirow{4}{*}{64} & 10 & 6.9321 & 2 & 29.4371 & 6.7851 & 29.4328 & 6.9156 & 29.4371 & 0.23859 & 1.88704 & 0 & 0.01461 \\
\hline & 20 & 7.7702 & 1 & 28.6214 & 7.8140 & 28.6214 & 7.7889 & 28.6215 & 0.24009 & 0.32225 & $3.49 \mathrm{E}-4$ & $3.49 \mathrm{E}-4$ \\
\hline & 50 & 8.9178 & 1 & 27.0425 & 9.3536 & 27.0422 & 9.0630 & 27.0426 & 1.60212 & 3.20644 & $3.70 \mathrm{E}-4$ & 0.00148 \\
\hline & 87.6 & 9.1681 & 1 & 26.1230 & 10.3570 & 26.1238 & 9.8773 & 26.1240 & 7.1801 & 4.85659 & 0.00383 & $7.66 \mathrm{E}-4$ \\
\hline \multirow{4}{*}{128} & 10 & 7.7582 & 1 & 35.1454 & 7.7654 & 35.1455 & 7.7855 & 35.1456 & 0.35065 & 0.25817 & $5.69 \mathrm{E}-4$ & $2.85 \mathrm{E}-4$ \\
\hline & 20 & 8.7504 & 1 & 34.5656 & 8.7942 & 34.5651 & 8.6771 & 34.5660 & 0.84475 & 1.34953 & 0.00116 & 0.0026 \\
\hline & 50 & 9.8980 & 1 & 33.0547 & 10.3339 & 33.0541 & 10.0017 & 33.0548 & 1.03682 & 3.32144 & $3.03 \mathrm{E}-4$ & 0.00212 \\
\hline & 87.6 & 10.1484 & 1 & 32.1414 & 11.3373 & 32.1423 & 10.8672 & 32.1425 & 6.6144 & 4.32586 & 0.00342 & $6.22 \mathrm{E}-4$ \\
\hline \multirow{4}{*}{256} & 10 & 8.7385 & 1 & 40.8260 & 8.7456 & 40.8257 & 8.6575 & 40.8278 & 0.9356 & 1.01761 & 0.00441 & 0.00514 \\
\hline & 20 & 9.7307 & 1 & 40.4983 & 9.7745 & 40.4972 & 9.5532 & 40.5005 & 1.85802 & 2.3165 & 0.00543 & 0.00815 \\
\hline & 50 & 10.8783 & 1 & 39.0661 & 11.3142 & 39.0649 & 10.8947 & 39.0661 & 0.15053 & 3.8505 & 0 & 0.00307 \\
\hline & 87.6 & 11.1286 & 1 & 38.1599 & 12.3175 & 38.1605 & 11.7766 & 38.1610 & 5.50244 & 4.59301 & 0.00288 & 0.00131 \\
\hline \multirow{4}{*}{512} & 10 & 9.5184 & 2 & 46.4865 & 9.7259 & 46.4769 & 9.5377 & 46.4866 & 0.20235 & 1.97322 & $2.15 \mathrm{E}-4$ & 0.02087 \\
\hline & 20 & 10.7109 & 1 & 46.4178 & 10.7548 & 46.4160 & 10.4320 & 46.4235 & 2.6735 & 3.09433 & 0.01228 & 0.01616 \\
\hline & 50 & 11.8585 & 1 & 45.0761 & 12.2944 & 45.0742 & 11.7802 & 45.0761 & 0.66467 & 4.36495 & 0 & 0.00422 \\
\hline & 87.6 & 12.1089 & 1 & 44.1783 & 13.2978 & 44.1785 & 12.6692 & 44.1791 & 4.42254 & 4.96164 & 0.00181 & 0.00136 \\
\hline \multirow{4}{*}{1024} & 10 & 10.4009 & 2 & 52.1269 & 10.7061 & 52.1058 & 10.4269 & 52.1271 & 0.24936 & 2.67769 & $3.84 \mathrm{E}-4$ & 0.04086 \\
\hline & 20 & 11.2799 & 2 & 52.3346 & 11.7350 & 52.3212 & 11.3175 & 52.3347 & 0.33223 & 3.68898 & $1.91 \mathrm{E}-4$ & 0.0258 \\
\hline & 50 & 12.8388 & 1 & 51.0844 & 13.2747 & 51.0818 & 12.6682 & 51.0847 & 1.34668 & 4.78758 & $5.87 \mathrm{E}-4$ & 0.00568 \\
\hline & 87.6 & 13.0892 & 1 & 50.1963 & 14.2780 & 50.1960 & 13.5612 & 50.1969 & 3.48052 & 5.28567 & 0.0012 & 0.00179 \\
\hline \multicolumn{2}{|c|}{ Average } & & 1.2 & & & & & & 1.996298 & 3.10695 & 0.001969 & 0.007862 \\
\hline
\end{tabular}
denotes errors related to the support region calculation and "sq" denotes values related to SQNR calculation.

TABLE I. SYSTEM PERFORMANCE $\left(x_{i n}=8\right)$. 
TABLE II. SYSTEM PERFORMANCE $\left(x_{i n}=10\right)$.

\begin{tabular}{|c|c|c|c|c|c|c|c|c|c|c|c|c|}
\hline \multirow[b]{2}{*}{$\mathbf{N}$} & \multirow[b]{2}{*}{$\mathbf{A}$} & \multicolumn{3}{|c|}{ Iterative procedure } & \multicolumn{2}{|c|}{ Closed-form solution } & \multicolumn{2}{|c|}{ Optimal solution } & \multicolumn{2}{|c|}{ Support rel. error } & \multicolumn{2}{|c|}{ SQNR rel. error } \\
\hline & & $x_{\max 1}$ & NoI & SQNR $_{1}[\mathrm{~dB}]$ & $x_{\max 2}$ & SQNR $_{2}[\mathrm{~dB}]$ & $x_{\max }$ & SQNR [dB] & $\delta_{1}{ }^{\mathrm{sp}}[\%]$ & $\delta_{2}$ sp $[\%]$ & $\delta_{1}$ sq $[\%]$ & $\delta_{2}$ sq $[\%]$ \\
\hline \multirow{4}{*}{64} & 10 & 6.9582 & 2 & 29.4367 & 6.5489 & 29.3992 & 6.9156 & 29.4371 & 0.616 & 5.3025 & 0.00136 & 0.12875 \\
\hline & 20 & 7.5403 & 1 & 28.6176 & 7.5431 & 28.6177 & 7.7889 & 28.6215 & 3.19172 & 3.15577 & 0.01363 & 0.01328 \\
\hline & 50 & 9.0215 & 1 & 27.0426 & 9.0572 & 27.0426 & 9.0630 & 27.0426 & 0.45791 & 0.064 & 0 & 0 \\
\hline & 87.6 & 10.0000 & 0 & 26.1240 & 10.0526 & 26.1240 & 9.8773 & 26.1240 & 1.24224 & 1.77478 & 0 & 0 \\
\hline \multirow{4}{*}{128} & 10 & 7.8184 & 2 & 35.1453 & 7.5291 & 35.1262 & 7.7855 & 35.1456 & 0.42258 & 3.2933 & $8.54 \mathrm{E}-4$ & 0.0552 \\
\hline & 20 & 8.6952 & 2 & 34.5660 & 8.5234 & 34.5643 & 8.6771 & 34.5660 & 0.2086 & 1.77133 & 0 & 0.00492 \\
\hline & 50 & 10.0000 & 0 & 33.0548 & 10.0375 & 33.0548 & 10.0017 & 33.0548 & 0.017 & 0.35794 & 0 & 0 \\
\hline & 87.6 & 10.0000 & 0 & 1407 & 11.0328 & & 10.8672 & & 7.97998 & 1.52385 & 0.0056 & 0 \\
\hline \multirow{4}{*}{256} & 10 & 8.6754 & 2 & 40.8277 & 8.5094 & 40.8212 & 8.6575 & 40.8278 & 0.20676 & 1.71066 & $2.45 \mathrm{E}-4$ & 0.01617 \\
\hline & 20 & 9.5008 & 1 & 40.5003 & 9.5037 & 40.5003 & 9.5532 & 40.5005 & 0.54851 & 0.51815 & $4.94 \mathrm{E}-4$ & $4.94 \mathrm{E}-4$ \\
\hline & 50 & 10.9821 & 1 & 39.0661 & 11.0177 & 39.0660 & 10.8947 & 39.0661 & 0.80222 & 1.12899 & 0 & $2.56 \mathrm{E}-4$ \\
\hline & 87.6 & 11.8482 & 1 & 38.1610 & 12.0131 & 38.1609 & 11.7766 & 38.1610 & 0.60799 & 2.00822 & 0 & $2.62 \mathrm{E}-4$ \\
\hline \multirow{4}{*}{512} & 10 & 9.4892 & 1 & 46.4859 & 9.4897 & 46.4859 & 9.5377 & 46.4866 & 0.50851 & 0.50327 & 0.00151 & 0.00151 \\
\hline & 20 & 10.4811 & 1 & 46.4234 & 10.4839 & 46.4233 & 10.4320 & 46.4235 & 0.47067 & 0.49751 & $2.15 \mathrm{E}-4$ & $4.31 \mathrm{E}-4$ \\
\hline & 50 & 11.9623 & 1 & 45.0759 & $\begin{array}{l}11.9980 \\
\end{array}$ & 45.0758 & 11.7802 & 45.0761 & 1.54581 & 1.84887 & $4.44 \mathrm{E}-4$ & $6.66 \mathrm{E}-4$ \\
\hline & 87.6 & 12.8285 & 1 & 44.1791 & 12.9933 & 44.1789 & 12.6692 & 44.1791 & 1.25738 & 2.55817 & 0 & $4.53 \mathrm{E}-4$ \\
\hline \multirow{4}{*}{1024} & 10 & 10.4694 & 1 & 52.1265 & 10.4699 & 52.1265 & 10.4269 & 52.1271 & 0.4076 & 0.41239 & 0.00115 & 0.00115 \\
\hline & 20 & 11.4613 & 1 & 52.3329 & 11.4642 & 52.3329 & 11.3175 & 52.3347 & 1.2706 & 1.29622 & 0.00344 & 0.00344 \\
\hline & 50 & 12.9426 & 1 & 51.0840 & 12.9783 & 51.0839 & 12.6682 & 51.0847 & 2.16605 & 2.44786 & 0.00137 & 0.00157 \\
\hline & 87.6 & 13.8087 & 1 & 50.1968 & 13.9736 & 50.1966 & 13.5612 & 50.1969 & 1.82506 & 3.04103 & $1.99 \mathrm{E}-4$ & $5.98 \mathrm{E}-4$ \\
\hline \multicolumn{2}{|c|}{ Average } & & 1.05 & & & & & & 1.28766 & 1.760741 & 0.001526 & 0.011458 \\
\hline
\end{tabular}

Data presented in Table I and Table II suggest that the required number of iterations $(\mathrm{NoI})$ for activating previously defined stop-criterion is lower in the case of $x_{i n}=10$. Moreover, calculated relative errors show that the average relative errors of the observed configurations are less in all cases for the optimal support determination using $x_{i n}=10$. Furthermore, the obtained results for SQNR are very close to the optimal solution which exploits numerically determined $x_{\max }$. The average relative errors between SQNR of the proposed system (both iteratively determined and exploiting closed-form solution) and the optimal solution are much less than $0.1 \%$ which is almost negligible.

Taking into consideration previous discussion, it could be summed up that the case of $x_{i n}=10$ provides more precise results for both iterative and closed-form determination. Based on that, we propose a closed-form solution for determination of the support region of semilogarithmic $A$-law quantizer for Laplacian source for a set of bit-rates $6 \mathrm{bits} / \mathrm{sample}-10 \mathrm{bits} / \mathrm{sample}$ (which corresponds to the set of 64-1024 quantization levels)

$$
x_{\max }^{\text {closed-form }} \approx \frac{1}{\sqrt{2}} \ln \frac{0.21 \times N^{2} A^{2}}{(1+\ln A)^{2}(1-\exp (-14.14 / A))} .
$$

\section{CONCLUSIONS}

In this paper, performance of semilogarithmic quantizer for Laplacian source was analysed. The analysis is performed for a set of values of the parameter $A(10,20,50$ and 87.6) and the set of bit-rates (6 bits/sample-10 bits/sample). The main contributions of the paper include proposing iterative solution for determining optimal support region as well as the asymptotic formula as a closed-form solution based on the analysis of the performance of iterative formula. The accuracy of the proposed solutions is compared with the optimal numerical solution using both relative errors between achieved SQNRs and support region values. It was demonstrated that the average relative errors between achieved SQNRs and the optimal solution are much less than $0.1 \%$, which can be considered as negligible and satisfactory for practical implementations.

\section{REFERENCES}

[1] S. A. Nandhini, S. Radha, "Compressed sensing based object detection and tracking system using measurement selection process for wireless visual sensor networks", Int. Conf. Wireless Communications, Signal Processing and Networking (WiSPNET), Chennai, 2016, pp. 1117-1122. DOI: 10.1109/WiSPNET.2016.7566310.

[2] Pulse code modulation (PCM) of voice frequencies, ITU-T Recommendation G.711, 1972.

[3] Yusuke Hiwasaki, Hitoshi Ohmuro, "ITU-T G. 711.1: Extending G 711 to higher-quality wideband speech", IEEE Communications Magazine, vol. 47, no. 10, pp. 110-116, 2009. DOI: 10.1109/MCOM.2009.5273817.

[4] N. S. Jayant, P. Noll, Digital Coding of Waveforms. Prentice Hall Pb, 1984.

[5] L. Hanzo, C. Somerville, J. Woodard, Voice and Audio Compression for Wireless Communications. John Wiley \& Sons - IEEE Press, 2007, pp. 11-28.

[6] S. Na, "On the support of fixed-rate minimum mean- squared error scalar quantizers for a Laplacian source", IEEE Trans. Inf. Theory, vol. 50, no. 5, pp. 937-944, 2004. DOI: 10.1109/TIT.2004.826686.

[7] S. Na, D. L. Neuhoff, "On the support of MSE-optimal, fixed-rate, scalar quantizers", IEEE Trans. Inf. Theory, vol. 47, no. 7, pp. 2972 2982, 2001. DOI: 10.1109/18.959274

[8] D. Aleksic, Z. Peric, J. Nikolic, "Support region determination of the quasilogarithmic quantizer for Laplacian source", Przeglad Elektrotechniczny, vol. 88, no. 7A, pp. 130-132, 2012.

[9] Z. Peric, M. Petkovic, J. Nikolic, A. Jovanovic, "Support region estimation of the product polar companded quantizer for Gaussian source", Signal Processing, vol. 143, pp. 140-145, 2018. DOI: 10.1016/j.sigpro.2017.08.024.

[10] Z. Peric, M. Dincic, D. Denic, A. Jocic, "Forward adaptive logarithmic quantizer with new lossless coding method for Laplacian source", Wireless Personal Communications, vol. 59, no. 4, pp. 625 641, 2011. DOI: 10.1007/s11277-010-9929-3.

[11] W. Cheney, D. Kincaid, Numerical Mathematics and Computing Thomson Higher Education, Belmont, 2008. 